\title{
Edge-glued wooden panel defect detection using deep learning
}

\author{
Lun-Chi Chen, et al. [full author details at the end of the article]
}

Received: 24 December 2020 / Accepted: 21 June 2021 / Published online: 31 January 2022

(C) The Author(s) 2022

\begin{abstract}
The wood-based furniture manufacturing industries prioritize quality of production to meet higher market demands. Identifying various types of edge-glued wooden panel defects are a challenge for a human worker or a camera. Several studies have shown that the detection of edge-glued defects with low, high, normal, overlong, short is identified but detection of residue and bluntness is highly challenging. Thus, the present model identifies defects of low, high, normal, overlong, short by computer vision and/or deep learning, whereas defects of residue and bluntness by deep learning based decide by pass for having better performance. The goal of this paper is to provide an improved defect detection solution for wood-based furniture manufacturing industries by process automation. Therefore, a system was designed that takes defect input images from a camera as raw image and laser-aligned image for defect detection of the edge-glued wooden panel. The process automation then performs computer visionbased image features extraction with deep learning for defect detection. The aim of this paper is to solve edge-glued defect detection problems by using design and implementation of edge-glued wooden defect detection, that can be stated as edge-glued wooden panel defect detection using deep learning (WDD-DL) for process automation by artificial intelligence and Automated Optical Inspection (AOI) consolidation. Possibly there exist several types of defects on the edges while edge-banding on the wooden panel in furniture manufacturing. Therefore, the scope is to achieve higher accuracy by raw image and laser-aligned image feature extraction using deep learning algorithms for final result defect classification in WDD-DL by AOI. The WDD-DL system uses Gabor, Harris corner, morphology, structured light detection and curvature calculation for pre-processing and InceptionResnetV2 Convolutional Neural Network algorithm to attain the best results. The applications of this work can be found in quality control of the furniture manufacturing industry for an edge, corner, joint defect detection of the wooden panels. The WDD-DL achieves best results as the precision, recall and F1 score are $0.97,0.90$ and 0.92, respectively. The experiments demonstrate higher accuracy achievement as compared to other methods with overkill and escape rate analysis. Ultimately, the discussion section provides an interesting experience sharing about the necessary factors for implementing the WDD-DL in real-time industrial operations.
\end{abstract}

Extended author information available on the last page of the article 


\section{Introduction}

Furniture manufacturing industries usually perform operations in the form of multiple independent stages. Each stage requires a particular operation that includes traditional ways of human skills based tasks, i.e., cutting, edge banding, border smoothing, etc. Process automation will help with quality control (Q.C.) checking for edge defect problems with better accuracy and less human dependency. The furniture manufacturing process can be detailed as initially importing raw material depending on the type of wood requirements. A wooden panel is then produced with proper cutting and polishing, thus a defect-free panel is passed on to the next stage. Selection of wooden panel is based on the respective furniture parts specification, which is then passed through an edge-glued banding machine. A traditional approach shows the oversized band is cut manually for border smoothing.

In practise, when the process is completed, the roughness on the edges is still present which compromises the quality as well as brand value. To improve the quality control process, WDD-DL provides a solution to identify the edge-glued defects and to show live defect screening as output. The conventional process of manufacturing involves only human workers in all the stages, which can be improved with the help of process automation for better and defect-free production. Applications include wood-based chairs, tables, closets, library racks, artwork, etc. Process automation is evaluated to be a better alternative in both cases of less infrastructure setup and less human worker dependency. In furniture manufacturing, the quality control requirements are satisfied by the WDD-DL edge defect detection system. Usually, during the quality control process, the detection of different types of edge defects is a challenge (Aleksi et al. 2019). The different types of edge-glued wooden panel defects can be given as high (the tape is higher than the surface of the panel), low (the tape is lower than the surface of the panel), bluntness (the tape is trim or has scrap on the edge), residue (visible glue marks), short (the tape is shorter than the length of the panel) and overlong (the tape is longer than the length of the panel). Practically, detecting the edge-glued wooden defects is different as compared to wooden edge defects (Aleksi et al. 2019; Wells et al. 2018) having edge knots, wane, split, pith, cracks, decays, etc.

The motivation for WDD-DL is how to achieve high accuracy for edge-glued wooden defect detection in the furniture industry? Chen et al. (2018) published a paper on edge detection based on machine vision, which describes the use of edge filter, canny operator and pixel-wise width calculation, but the results provided for accuracy were quite inefficient. Therefore, a necessity to design a new approach was realized that can provide higher accuracy and help in industrial process automation. The background for WDD-DL can be referenced by various edge detector, filters and classifiers of visual-based defect detection methods in manufacturing industries (Czimmermann et al. 2020). The Harris corner is a wellknown computer vision algorithm that calculates differential of the corner score with respect to direction. It has a high edge and corner distinguishing capability. The process involved is colour to gray-scale conversion for enhancing processing 
speed, spatial derivative calculation, structure tensor setup, Harris response calculation and non-maximum suppression for picking up optimal values. The Gabor filter is a linear filter used for texture analysis, that can be described as analyzing frequency content within an image's specific region of analysis. In the case of Local Binary Pattern (LBP), it requires fewer computations and quite an efficient filter for defect detection. As the surface image has low-rank structure, defects within them will be quite hard to detect. So, entity sparsity pursuit model is used to detect such defects and resolve it based on LBP (Wang et al. 2019). Micro defects present within OLED panels are detected using modified LBP and SVM classifier for better performance (Sindagi and Srivastava 2015). Edge features extraction is used for many defect detection approaches that have a criterion for edge length, strength and calculating edge neighbours having grey level pixel values for distribution (Wen and Xia 1999). In case of processing, texture elements in spatial locations, also known as texture primitives are usually individual pixel or grey-scale regions. To improve segmentation in the texture based defects for fabric processing, Cao et al. (2017) implemented subspace segmentation modelling using local priori algorithm. Morphological based defects detection used to avoid fatal defects before steel surface cracks can occur is presented by Yun et al. (2019). An exhaustive dynamic encoding algorithm is used for searching such defects by optimizing structural elements. The WDD-DL edge defects detection process consists of checking defects in the raw image and laser-aligned imagebased features using detail inspection by deep learning. Multi-sensor (Wells et al. 2018) systems have been dominating the industrial detection environment for a long time. The use of line laser and video camera constitutes a distinct approach, that is applied to smart manufacturing (Contreras Masse 2019) for process automation of design and implementation of wooden panel edge defect detection using deep learning algorithms (WDD-DL). Considering the case of defect detection approach, segmentation (global) and non-segmentation (local) approaches were used for defect object detection and non-overlapping rectangular defect regain parts are used, respectively. The WDD-DL uses a non-segmentation approach, to evaluate different detection algorithms and provide an efficient result. The two-stage system uses different detection algorithms consisting of raw image and laser-aligned image-based features using deep learning. A sequence of operations is completed by process automation and will help manual workers to watch a live screening of edge defects on the wooden panel during the manufacturing process. In short, WDD-DL uses multi-sensor, two-stage system for process automation by improving the quality of work. Ultimately, a defect-free and parallel manufacturing process automation is accomplished. In WDD-DL, the research gap can be given about the edge-glued defect detection, which has hardly been presented before. Most of the defect detection process focuses on panel or surface-based defects, so the present model is exclusively built for improving the edge defect detection process. The WDD-DL research is rationale because it provides a new two-stage approach to solve edge-glued defect detection problem. This approach is practical for the furniture industry in the quality control process. Table 1 shows a comparison of different defect detection models in detail. 


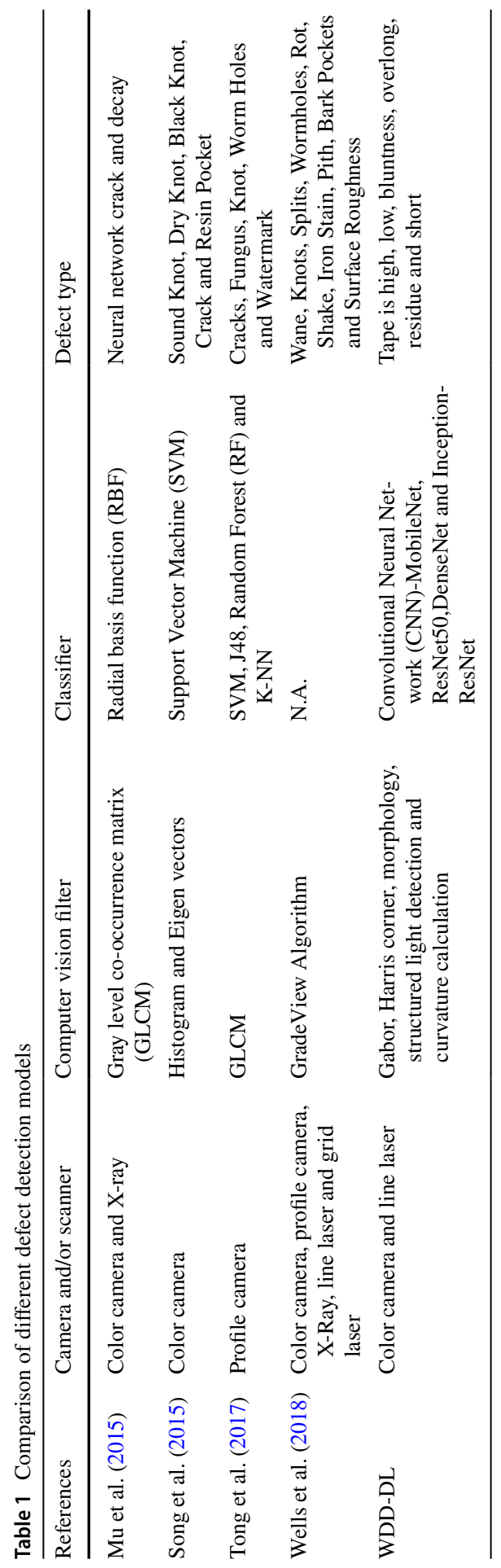


Considering the results presented by most of the automation systems (Aleksi et al. 2019; Czimmermann et al. 2020), they are inefficient in accurately identifying edge defects on the wooden panel. Efforts are made to increase the quality control, but lack of high accuracy edge defect detection is the limitation of the literature work. Therefore, to achieve higher accuracy in the defect detection process, WDD-DL consists of a two-stage model that performs extraction of image features from the raw image and laser-aligned image using deep classification-based evaluation. The aim of this study is to solve edge defect detection problems, which can be stated as edge-glued wooden panel defect detection using deep learning (WDDDL) for process automation. The scope is to achieve higher accuracy by image and laser feature extraction using deep learning algorithms for final result evaluation in WDD-DL. Table S1 in Electronic Supplementary Material provides a comparison of different defect detection models for the industrial quality control.

The WDD-DL objectives are in detail as follows:

- Solving the wooden panel edge defect detection using AOI-based process automation: AOI-based process automation is considered as a crucial factor in wooden panel edge defect detection. Previously, trained workers were assigned in every manufacturing stage to identify wooden panel defects and remove them separately. This manual process was not effective enough as they have missed many edge defect errors quite frequently. To overcome such problems, process automation is provided to identify defects in the raw image and laser-aligned image features by the industrial camera for classification. Henceforth, various popular CNN models are used here for deep learningbased classification.

- Real-time display of edge defects on the live screen: Most of the machine learning and deep learning methods require high processing time for classification that form a bottleneck for live screen display of edge defects. However, there exist some simplified filters based on a rule-based system to solve such issues. For efficient processing, the wooden panel edge defects must be identified in real-time for shorter or long edge defects with both for straight edge and corner of edge. So, WDD-DL solves the challenge of detecting different edge defect types by multi-sensor and two-stage process in real-time. The real-time live screening of edge defects is processed by using a raw image and laser-aligned image features, where deep learning classification is optional. Different types of edge-glued wooden panel defects can be identified by raw image and laser-aligned image features using deep classification model.

- Deploying the model in the live manufacturing environment of the furniture industry: Even though the experiments will demonstrate the accuracy and performance of the model in various scenarios, a discussion with the manufacturing workers will help to share detailed experiences as presented in the discussion section. Henceforth, a live production environment will help us to understand the practical aspects of the manufacturing scenario. 


\section{Literature survey}

Recently, process automation within the furniture manufacturing industries was performed for increasing quality, productivity and decreasing human-related errors. Even though the traditional process automation was performed involving segmentation (geometrical features) and non-segmentation (tonal and textual features) approach, they were not found efficient for wooden panel edge/corner defect detection because of their low defect detection accuracy. Therefore, the present paper proposes the WDD-DL two-stage approach combining filters and deep learning, involving morphological and curvature calculation of edges in images with laser information as a new two-stage approach to achieve higher accuracy for the quality control process. Some recent studies are shown in Fig. 1. Defect control strategy analysis consists of defect detection in manufacturing, which includes statistics, machine and deep learning.

Several statistical feature extractions based models for wooden defect detection including Linear Discriminant Analysis (LDA), Local Binary Patterns (LBP), Gray Level Dependence Matrix (GLDM), Partial Least Squares (PLS), GradeView algorithm, etc., are given below. The sawn wooden board-based texture used for feature extraction and defect detection is demonstrated by Aleksi et al. (2019). The modelling is performed using feature selection by statistical feature extraction, texture defect detection and signal to noise ratio calculation. The automated grading system for hardwood lumber defect detection is presented by Wells et al. (2018). In nine different types of hardwood lumber, the defect detection model consists of board scanning with Microtec Goldeneye 300 multi-sensor-quality scanner, and data were analyzed with the Purdue GradeView algorithm. The use of local binary pattern variants for wood defect image classification was performed by Rahiddin et al. (2020). The basic and variants of the LBP feature set are constructed, from a stage of feature extraction processes with the Basic LBP, Rotation Invariant LBP, Uniform LBP, and Rotation Invariant Uniform LBP.

The wood defect classes were further evaluated with the use of ANN, KNN and J48 decision tree classifiers. Classification of wood defects for texture feature

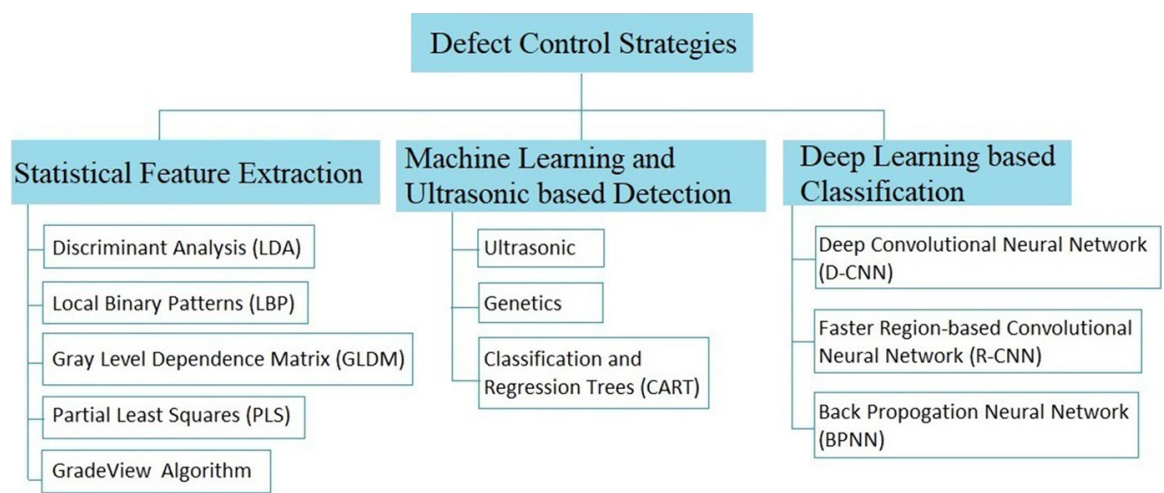

Fig. 1 Defect control strategy analysis 
analysis is demonstrated by Abdullah et al. (2020). The extraction and analysis of features are based on statistical texture in wood defects images. Feature extraction using the Grey Level Dependence Matrix (GLDM) and feature analysis were executed to investigate the appropriate displacement and quantization parameters that could significantly classify wood defects. Timber defect classification by multivariate texture descriptor performance evaluation is demonstrated by Hashim et al. (2016). This paper presents a performance evaluation of texture features based on orientation independent Grey Level Dependence Matrix (GLDM) for the classification of timber defects and clear wood. A series of processes including feature extraction and feature analysis was implemented to facilitate data understanding in order to construct a good feature. Wood defects soft measurement by feature fusion of LDA and the compressed image sensor is presented by Li et al. (2017). Linear Discriminant Analysis (LDA) algorithm was used to integrate these features and reduce their dimensions. Features after fusion were used to construct a data dictionary and a compressed sensor was designed to recognize the wood defects types. Appearance lumber-based resin defect detection by 2D NIR spectroscopy is demonstrated by Thumm and Riddell (2017). Resinous defects found on the finished products of a barge's boards and weatherboards, can cause resin bleed and resin show-through, which is a discoloration of the paint layer above the resin feature. Several efforts are made to eliminate resinous wood via manual and automated grading systems. 2D histogram-based classification of wood defects using Local Binary Differential Excitation Pattern and LBP is presented by Li et al. (2019). A classification algorithm based on LBP and local binary differential excitation pattern is presented for the classification of the crack and the linear mineral line on the surface of the birch veneer. The local binary differential excitation pattern (LB-DEP) is a texture description model proposed in this paper, which is generated by the combination of LBP and Weber's Law. The 2D histogram is used to classify the defects with Euclidean distance classifier.

In case of defect detection using ultrasonic testing and machine learning model, they can be further described by ultrasonic, genetic and classification and regression trees (CART). Wood and wooden products testing by using air-coupled ultrasonic technologies is presented as a review by Fang et al. (2017). Air coupled ultrasonic (ACU) testing is a contactless ultrasonic measurement method used for material characterization. To not contaminate during the testing processes by coupling agents utilized in conventional ultrasonic testing, correlations between the ACU parameters (amplitude, velocity, and spectrum) and the wood properties (i.e., density, moisture content, strength, and stiffness) as well as the wood defects (knots, cracks, decay, insect damage, and delamination) are inspected. Wood hole defects feature extraction by wavelet-based ultrasonic testing is presented by Yang and Yu (2017). Wavelet energy moment can reflect the distribution of energy along the time axis, the amount of energy in each frequency band and later extract the energy distribution characteristics of signals in each frequency band. Therefore, wavelet energy moment can replace the wavelet frequency band energy and constitute wood defect feature vectors by PCA and feature vectors.

Deep learning methods including CNN, R-CNN, GAN-CNN, BPNN in computer vision are a preferred method for the recent studies, which is given as follows. 
Wooden defect feature extraction and detection by using deep convolutional neural networks is demonstrated by $\mathrm{He}$ et al. (2020). A laser scanner is used through a deep convolutional neural network (D-CNN) and TensorFlow to train the network, which consists of an input layer, four convolutional layers, four max-pooling layers, three fully-connected layers, a softmax layer, and an output layer. Additional configurations involve dropout, L2 regularization, and data augmentation to avoid the overfitting problem. Wood defect feature extraction and classification using BP neural network, wavelet moment and $\mathrm{Hu}$ moment are demonstrated by $\mathrm{He}$ et al. (2020). Five features of training samples are extracted from the area, roundness and circumference, which are represented by $(\mathrm{x} 1, \mathrm{x} 2, \mathrm{x} 3)$ to form a three-dimensional vector, so 150 test samples form a $150 * 3$ matrix. Characteristics of wavelet energy, the principle and characteristics of $\mathrm{Hu}$ moment invariant and extraction of wavelet moment features are utilized. Wood veneer surface defect identification using faster region CNN and transfer learning is presented by Urbonas et al. (2019). A faster region-based convolutional neural network (Faster R-CNN) for the identification of defects on wood veneer surface texture is presented. To improve the results, pre-trained AlexNet, VGG16, BNInception, and ResNet152 neural network models for transfer learning are used. Lumber tasks classification by using deep learning is demonstrated by $\mathrm{Hu}$ et al. (2019). The four datasets used in this work were manually marked as lumber defects, wood textures and lumbers by experts with configured 80:20 ratios for training and testing. They investigate variations of deep learning strategies based on ResNet18 for classification of lumber images. Surface defect inspection by using weakly supervised learning from deep convolutional networks is presented by Chen et al. (2020). A novel end-to-end CNN architecture integrating the robust classifier and spatial attention module is proposed to enhance defect feature representation ability for classification accuracy. Spatial attention class activation map (SA-CAM) is added to improve segmentation adaptability by generating more accurate heat map. Defect recognition by using deep learning for multi-level fusion of information is demonstrated by Gao et al. (2019). A three-level Gaussian pyramid is introduced to generate the multi-level information of the defect, for model training. Three VGG16 networks are built to learn the information, and the outputs are fused for the final recognition result. Wood defect identification and location by using convolutional neural networks is presented by He et al. (2019a). In Mix-FCN, filter weights were initialized during training from the trained VGG16 model, whereas weights of VGG16 were learned from the dataset 1 and their system model was trained, validated and tested on dataset 2. Overall classification accuracy (OCA), pixel accuracy (PA), mean intersection over union, detection rate, missing alarm, false alarm rate, and precision were evaluation indicators. Surface defect classification using image segmentation by convex optimization is presented by Chang et al. (2018). The convex optimization (CO) with different weights is used as a pretreatment method for smoothing and the Otsu segmentation method to obtain the target defect area images. Structural similarity (SSIM) results between the original image and defect image were calculated to evaluate the performance of segmentation with different convex optimization weights. The geometric and intensity features of defects were extracted before constructing a classification and regression tree (CART) classifier. 
Ultimately, the following achievements of WDD-DL are summarized as compared to the limitations observed in the above literature survey:

1. WDD-DL uses both segmentation and non-segmentation approach, to decide which approach suits better with different types of edge-glued wooden panel defects as verified by the experiments.

2. The use of hybrid approach provides a better accuracy, which was rarely utilized for implementation. Moreover, it performs better than the statistical and computer vision filters to detect various types of defects in the industrial process.

3. The use of multi-sensor, two-stage design approaches yields better performance.

4. The algorithms are defined properly in pseudo-code and are quite practical to implement.

\section{Methodology}

In the methodology section, the workflow process pre-requirements are presented that enable the workflow in the furniture manufacturing industry. The WDD-DL operational process is described as a workflow model given below:

- Resource Requirement: The manufacturing process must plan for detailed resource requirements. The resources include input raw material, types of material, processing machinery, human workers, etc. In addition, all the resources must be available, so as to be ready before the manufacturing process. An appropriate schedule plan ensures smooth initialization and completion of the workflow.

- Process Planning: Planning specifies the synchronization of process steps that are required to be implemented. A proper plan will ensure fixed operations with optimal steps. Details of the machine usage, resource availability, human worker task assignment, etc., are crucial factors of process planning. Each task performed with the respective resources will be specified with a timing diagram/chart for efficient operations.

- Quality Control: The grading of resources, evaluation, outcome is the measure of quality control. Therefore, the wooden panel types, defect types, wastage in production, accuracy in production, and evaluation by the international quality standard are performed. For manual workers, their skill training, past experience, performance are also considered. In short, the quality control process helps to reduce time, wastage and schedule resources for better performance.

The approached system WDD-DL, as shown in Fig. 2, is presented in four major categories: The input provided to the system, AOI-based process automation that automates the manufacturing system, filtering and classification using deep learning and real-time defect detection for the manufacturing process.

(Note: Raw image is input to the computer vision algorithm, whereas laseraligned image is input for CNN.) 


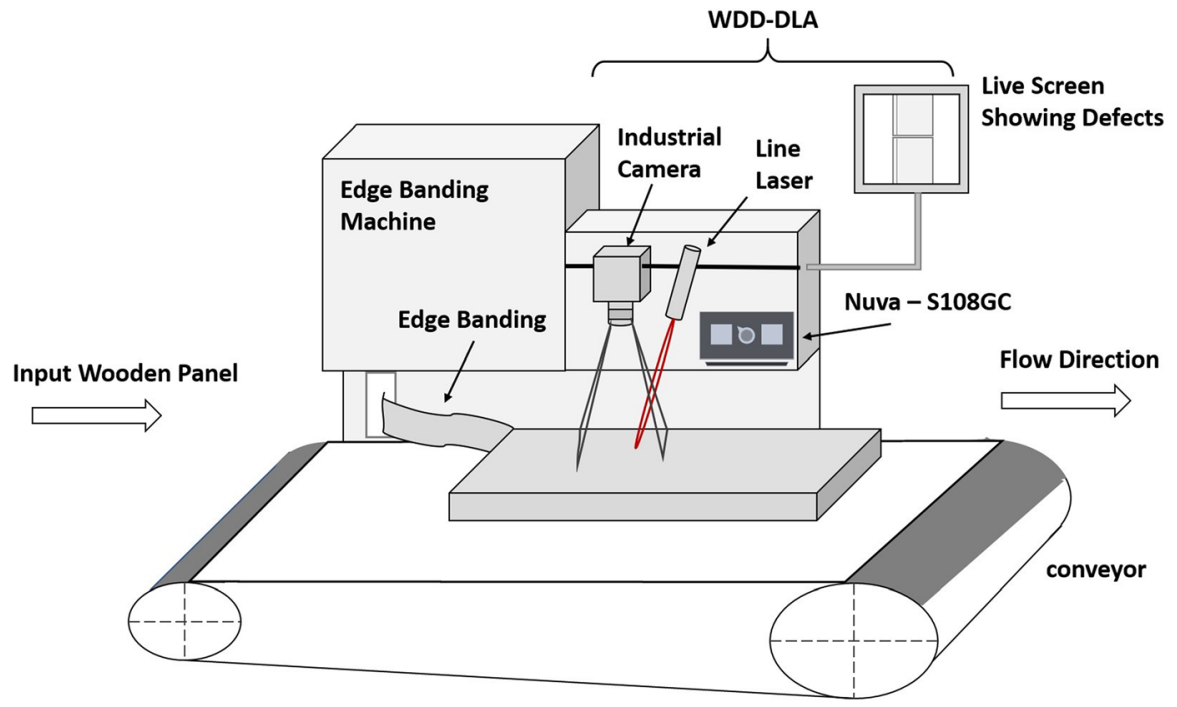

Fig. 2 System model

\section{WDD-DL system model}

1. Input to the System: The input provided to the system consists of a specific type of wooden panel, to detect the edge-glued defects on it. In addition, the wood panel exact size, positioning and placement on the conveyor should be determined.

2. AOI-based Process Automation: After the input is given to the system in the form of a wooden panel, the defect detection process is known to be initialized. The AOI-based process automation consists of controlling all of the hardware and software within the WDD-DL. At first, when the input is provided in the form of a wooden panel on the conveyor, the edge banding on the wooden panel is performed by the edge banding machine, as shown in Fig. 2. The WDD-DL is evaluated for detecting the accuracy of band placement on the edge-glued wooden panel for the defect types identification. The identification of the defect will be based on raw image, line laser-based image processing for computer vision with the help of industrial camera having set the focal length and field of view (FOV), which is processed by a local Nuvo-8108GC computer. Evaluating the results is based on the environment having a camera, line laser, a combination of lenses and its holder. Ultimately, the objective is to achieve real time live defect detection on the wooden panel at the end of the conveyor as output.

3. Computer Vision-based Filtering and Deep Learning based Classification: The filtering is performed on the raw image and on line laser image by using computer vision algorithms so that the defects can be identified on the edge-glued wooden panel after the edge banding process. Figures 3 and 4 are used to describe the wooden defect detection system with the flowchart and its functions, respectively. The WDD-DL process automation inspects the raw image and laser image-based region-of-interest (ROI) for filtering and classification. In the beginning, the 


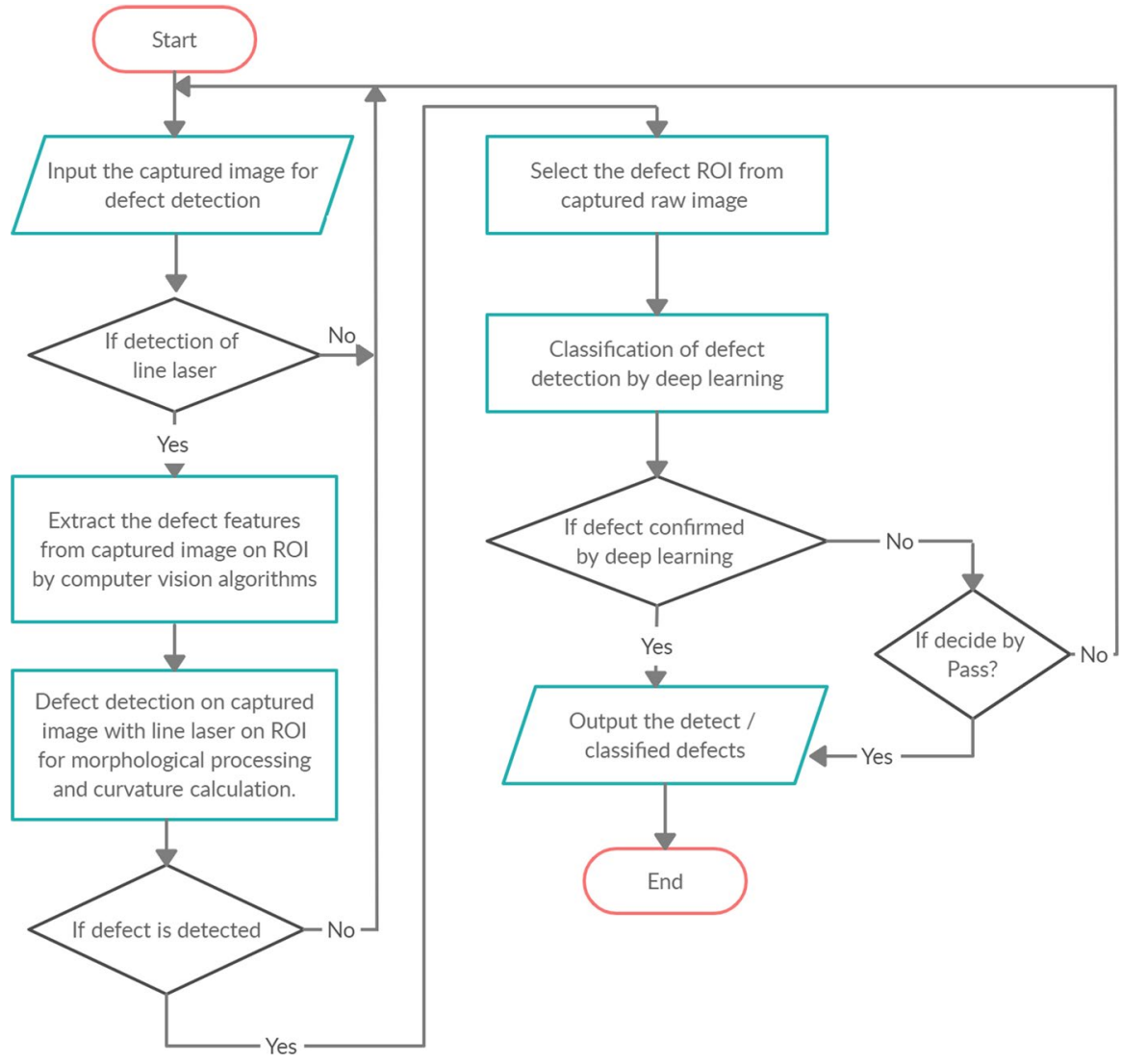

Fig. 3 Flowchart for the system model

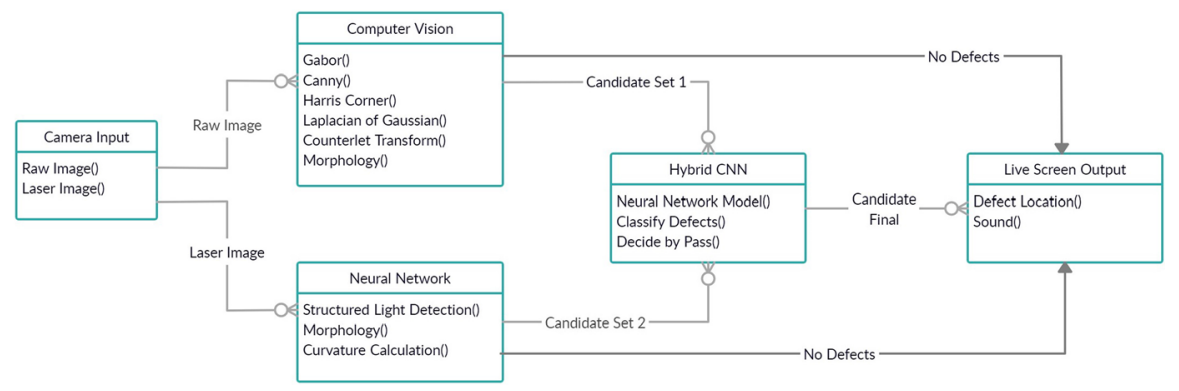

Fig. 4 Functional model

image is captured for a wooden panel which needs to be inspected and is given as input to the system. The system then checks whether the line laser mark is present within the image, if not present then the system is required to recapture the image. The absence of a line laser in the captured image may be due to weather 
conditions, camera blurring, noise or other technical problems. The defect features are then extracted from the raw image using k-means with contours, comparing pixel distribution, evaluating pixel after applying the threshold, means denoising, Harris corner, local binary patterns (LBP), a histogram of oriented gradients (HOG), wavelet-based contourlet transform, Gabor/convert scale edge detection based on using Canny, Sobel, Prewitt, Roberts and Laplace. Ultimately, WDD-DL has better defect detection achieved by Harris corner and Gabor filter based on the results demonstrated in the experiment section. Whereas, the types of edge-glued wooden defects present within WDD-DL are high, low, bluntness, residue, short and overlong. For the line laser image, the features are extracted using morphological processing and curvature calculation. After the above two steps of defect feature extraction, if the present defect is detected by the camera, then the defect ROI raw image is further passed for classification using deep learning. If no defect is detected, then the camera continues to take new images from the other parts of the edge-glued wooden panel based on the speed motion of the conveyor. The deep learning classification models used are ResNet50, MobileNetV2, DenseNet201 and InceptionResNetV2. If the defect is classified by the deep learning model, then the defect area is highlighted on the real-time live output screen, so that the human worker can notice it and can place that particular wooden panel separately as an exception. The defect types are given as low (L), high $(\mathrm{H})$, normal $(\mathrm{N})$, overlong $(\mathrm{O})$ and short $(\mathrm{S})$, which if detected by computer vision then the results are declared even if unclassified by deep learning. In case, the defect is not classified by the deep learning classifier, even after confirmed by the computer vision algorithm, then it has to be confirmed by pass. For the case of defect type residue (R) and bluntness (B) from captured image input with line laser, when the control is in decide by pass function and if detected by computer vision but not classified by deep learning, then it will use the result by deep learning instead. If deep learning detects the defect, then it will output the defect type without decide by pass. The functional model as shown in Fig. 4. presents functions used within different stages of WDD-DL system.

4. Real-Time Defect Detection: The live screen is used to display the wooden panel passing on the conveyer for quality inspection. The WDD-DL system helps to identify edge defects in real-time and displays the defects on the edge-glued wooden panel with the banding errors by highlighting the prone area. Therefore, an alert is raised for helping the machine operator to notice the defect with the beep sound, as passing the quality control checkpoint in that stage. Real-time processing eases the manual work of missing the defect detection and ensures high-quality checking and separating out the defective items.

\section{Mathematical model}

For the effective corner detection of better invariance including scale, rotation, image noise and illumination variance, Harris corner detector is utilized (Derpanis 2004). In this case, signals use the local function of auto-correlation which identifies local changes by a measure for shifting patches in various directions. In Eq. (1), the 
auto-correlation function can be given with the points $(\mathrm{x}, \mathrm{y})$ and the shift $(\Delta \mathrm{x}, \Delta \mathrm{y})$ as,

$$
c(x, y)=\sum_{w}\left[I\left(x_{i}, y_{i}\right)-I\left(x_{i}+\Delta x, y_{i}+\Delta y\right)\right]^{2}
$$

In Equation $(1), \mathrm{I}(.,$.$) is image function and \left(x_{i}, y_{i}\right)$ is centered $(\mathrm{x}, \mathrm{y})$ points of the $\mathrm{W}$ (Gaussian) window. The Taylor expansion approximation for the shifted image can be given in first-order term as:

$$
\begin{aligned}
& I\left(x_{i}+\Delta x, y_{i}+\Delta y\right) \approx \\
& I\left(x_{i}, y_{i}\right)+\left[I_{x}\left(x_{i}, y_{i}\right) I_{y}\left(x_{i}, y_{i}\right)\right]\left[\frac{\Delta x}{\Delta y}\right]
\end{aligned}
$$

In Eq. (2), $I_{x}\left(.\right.$, .) and $I_{y}(.,$.$) is used to denote (x,y) partial derivatives, respectively.$

$$
c(x, y)=[\Delta x \Delta y] C(x, y)\left[\frac{\Delta x}{\Delta y}\right]
$$

Equation (3) is obtained by substituting Eq. (2) into (1). Here, c(x,y) is used to denote capturing of local neighbourhood intensity structure. Let the matrix $\mathrm{c}(\mathrm{x}, \mathrm{y})$ have eigenvalues $\lambda_{1}, \lambda_{2}$ forming the description for rotational invariant. When both $\lambda_{1}, \lambda_{2}$ are low, high and opposite then it represents constant intensity, corner and edge, respectively. Another corner detection algorithm, Gabor function given in Eq. (4) is used by Gaussian function as complex exponentially modulated operation (Kumar and Pang 2002a). A non-orthogonal basis set is formed by complete Gabor function and in 2-D plane, its impulse response can be given as:

$$
g(x, y)=\left(\frac{1}{2 \pi \sigma_{x} \sigma_{y}}\right) \exp \left[-\frac{1}{2}\left(\frac{x^{2}}{\sigma_{x}^{2}}+\frac{y^{2}}{\sigma_{y}^{2}}\right)+2 \pi j w x\right]
$$

The Gabor function's radial frequency is denoted by $u_{o}$. The (x, y) axis with Gaussian envelope can be given as $\sigma_{x}, \sigma_{y}$ space constants. Gabor functions behave as a bandpass filter in the frequency domain and $\mathrm{f}(\mathrm{x}, \mathrm{y})$ Fourier transform is given as:

$$
G(u, v)=\exp -\frac{1}{2}\left[\frac{(u-W)^{2}}{\sigma_{u}^{2}}-\frac{v^{2}}{\sigma_{v}^{2}}\right]
$$

In Eq. (5), $\sigma_{u}=\frac{1}{2} \pi \sigma_{x}$ and $\sigma_{v}=\frac{1}{2} \pi \sigma_{y}$. The learning for deep residuals can be given as a subset of stacked layer mapping as $\mathrm{H}(\mathrm{x})$, where prior layers' input is x. Assuming asymptotic approximations of several nonlinear layers of complicated functions then it is also equivalent to residual function, i.e., $\mathrm{H}(\mathrm{x})-\mathrm{x}$, having the same dimensions for input and output. So, $\mathrm{F}(\mathrm{x}):=\mathrm{H}(\mathrm{x})-\mathrm{x}$ can be given as distinct for different functions. The training error for deep models (He et al. 2016) is equivalent to shallow models when identity mapping is constructed from added layers. The difficulties given by solvers for mapping identity to deep nonlinear layers are found to suffer degradation. For the optimal identity mappings with the reformulation of residual learning, the solver makes the weight tune to zero for identity mappings of the deep nonlinear layers. Reformulation helps in the case of precondition problems having less optimal identity mapping in reality. It is considered to be efficient for searching permutations referring to identity mapping, when the closeness of identity mapping 
is to optimal function instead of new function learning. Every subset of stacked layers adapts to residual learning. The building block is given as

$$
y=\mathcal{F}\left(x,\left\{W_{i}\right\}\right)+x
$$

Here, we consider the input and output as $\mathrm{x}$ and y layers, respectively. In Eq. (6), the learning for residual mapping can be given by $\mathcal{F}\left(x,\left\{W_{i}\right\}\right)$ function. For two layers, $\mathcal{F}=W_{2 \sigma}\left(W_{1} x\right)$ where ReLU is given as $\sigma$ and notation simplifying by omitting biases. The element-wise addition and shortcut connection are $\mathcal{F}+\mathrm{x}$ operation, $\sigma(y)$ is second nonlinearity adapted after addition. The shortcut connections use $W_{s}$ as a linear projection for dimension matching, when $\mathrm{x}$ and $\mathcal{F}$ dimensions are not equal.

$$
y=\mathcal{F}\left(x,\left\{W_{i}\right\}\right)+W_{s} x
$$

$W_{s}$ square matrix can be used in Eq. (7). Here, $\mathcal{F}$ is flexible as a residual function. The notations given above can be applied to both fully connected layer and convolutional layer, where deep convolution layer can be given by $y=\mathcal{F}\left(x,\left\{W_{i}\right\}\right)$. The feature map uses channel by channel element-wise addition. For training high definition mini-batch SGD (Akiba et al. 2017), the SGD and RMSprop are used to represent a simplified combination of momentum as an update rule.

In Inception Resnet (Zhang and Davison 2019), the extracted features have detailed information, achieving higher accuracy by the classifier. The features are trained for the robust classifier to account quantitatively for the conditional and marginal distribution having relative importance with the function, as distribution alignment is dynamic. Manifold learning is used to present learned feature alignment given by Manifold Embedded Distribution Alignment (MEDA). The process can be given as: (a) manifold-based feature learning, (b) the conditional and marginal distribution of data is aligned-based on dynamic distribution and (c) the estimated parameters are used for classified labels updating.

In modified distribution alignment, original features are preferred over the use of GFK model features. It can be justified as: (a) the GFK model undetermined dimensionality is avoided by the original features information and (b) the classification problem can obtain required detailed information from the Inception Resnet (IR) feature extraction.

\section{Algorithms}

This section presents algorithms with detail description used to carry out two-stage operation process of WDD-DLA that includes computer vision-based defect detection, neural network-based defect detection and hybrid algorithm for final results using computer vision and neural network-based defect detection.

The Algorithm 1. AOI for computer vision-based defect detection is given in the form of pseudo-code. In step 1, the input is given to the algorithm using a camera. The input image consists of both raw image and laser-aligned image. The raw image does not consider the presence of line laser, whereas the laser-aligned image requires line laser mark for its processing. In step 2, the output of the algorithm is given as candidateSet 1 , which consists of computer vision-based filtered defects 
given as C. In step 3, the candidateSet 1 is initialized to NULL. In step 4, the If condition checks whether the input image given to the algorithm is able to detect the Region of Interest (ROI) in both the raw and laser-aligned image. In step 5, if the previous condition is true, then morphological ROI feature ( $\mathrm{fm}$ ) is calculated from the laser-aligned image to enhance laser features. In step 6, a new loop is started for each laser feature taken from the 3D matrix of laser image to calculate pixel depth difference having range 1 to $\mathrm{k}$, where $\mathrm{k}$ is given as all of the non-segmented defects detected from the laser image. In step 7, the depth curvature $\left(\right.$ Depth $\left._{C u r}\right)$ is calculated

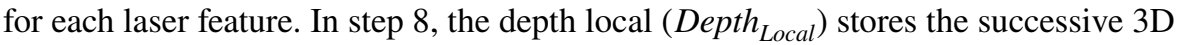
matrix difference from 1 to $\mathrm{k}$ given as adjacency difference. In step 9, a new if condition checks whether the depth locally calculated is greater than the tolerance limit and depth curvature is within the curvature threshold limit. In step 10, if the above condition is true then candidateSet 1 stores the classified defects that can either hold one of the values from high, low and overlong.

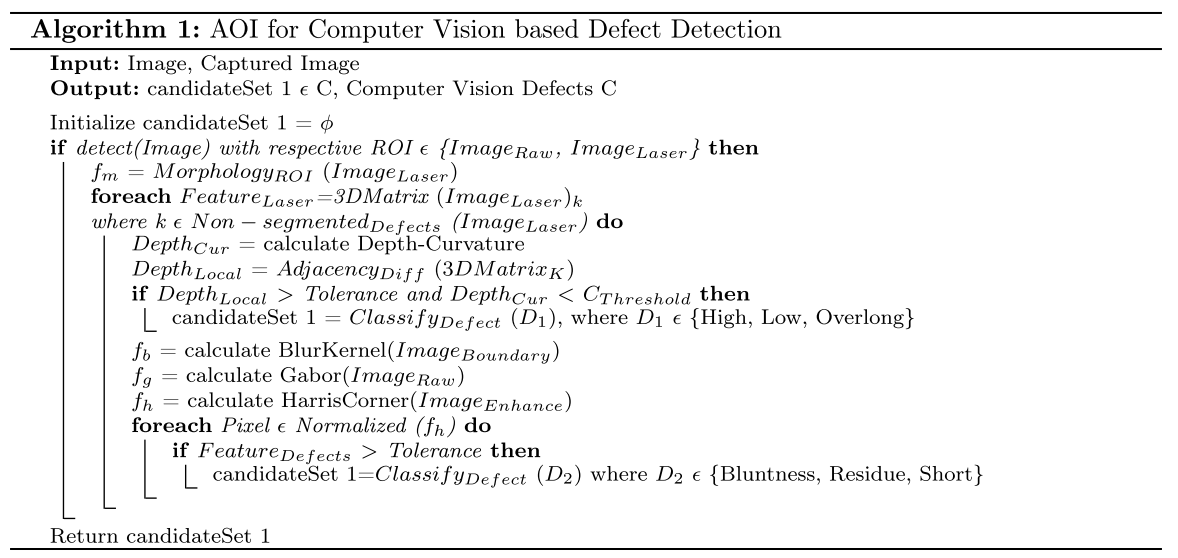

In step 11 , the blur kernel $\left(f_{b}\right)$ stores the calculated blur sharp boundary. In step 12 , the Gabor filter $\left(f_{g}\right)$ stores the edge texture analysis features calculated from the raw image. In step 13, the Harris-corner filter $\left(f_{h}\right)$ is used to store blunt boundary features from the enhanced edge banding featured image. In step 14, inherited for loop checks defect features for each pixel of normalized fh. In step 15, if condition checks whether the defect features within that pixel are higher than the tolerance limit. In step 16, if the above condition is true then candidateSet 1 stores the classified defects as bluntness, residue and short. In step 17, finally after processing all the pixels within the target image, the defect features computed by computer vision are returned as candidateSet 1 .

The Algorithm 2 pseudo-code given above is AOI for neural network-based defect calculation in the pseudo-code form. In step 1, the input (Image) to the algorithm is given by a camera for defect detection. In step 2, the output is given as candidateSet 2 , which contains the defects detected by the neural network on the input image. In step 3 , the neural network confidence $\left(N N_{\text {Conf }}\right)$ is given by the model predicted 
confidence. In step 4, the candidate set 2 is initialized by setting it to NULL. In step 5 , the input image captured by the camera may have different dimensions depending on the camera picture resolution, so the image is resized to the standard neural network input image dimensions. In step 6, after providing the resize image as input to the neural network, the output is provided in the form of classified defects type $\left(\right.$ Classify $\left._{\text {Defect }}\right)$ and neural network confidence $\left(N N_{\text {Conf }}\right)$ about that classification. In step 7, candidateSet 2 is added with the output of the neural network having ClassifyDefects and $N N_{\text {Conf }}$ as the format. In step 8, the output is returned by the algorithm as candidateSet 2 for the defect features computed by neural network.



The Algorithm 3 given is a computer vision and neural network hybrid algorithm for the final defect result detection presented in the pseudo-code form. In step 1, the input given to the algorithm is the captured image from the camera. In step 2, candidateSet 1 is used which stores the AOI-based computer vision detected defects. In step 3, candidateSet 2 is used which stores the AOI-based neural network detected defects. In step 4 , the $N N_{\text {Conf }}$ is used to store a prediction, specifically neural network model confidence about the detected defect. In step 5, the output candidate $_{\text {Final }}$ determines whether the output type will be predicted by computer vision defect or neural network defect or by combining both hybrid. In step 6, the result candidate $_{\text {Final }}$ is initialized to NULL. In step 7, the if condition checks that whether candidateSet 1 is a subset of either having a defect in D1 set defect type low, high, normal, overlong and short. In step 8, if the previous condition is true then a new inherited If condition checks whether $N N_{\text {Conf }}$ is greater than or equal to $80 \%$ and none of the candidateSet 1 values match with the candidateSet 2 values. In step 9, when the previous two conditions are true then the result candidateFinal is assigned the values by adding candidateSet 1 and candidateSet 2 . In step 10, if step 8 is not true then the result candidate $_{\text {Final }}$ in step 11 is assigned only the values from candidateSet 1 . Step 12 checks if the candidateSet 1 is a subset of value from D2 set having defect type as residue or bluntness. Step 13 verifies if the previous condition is true and $N N_{\text {Conf }}$ is greater than or equal to $80 \%$. In step 14, when the previous two conditions are true then candidate $_{\text {Final }}$ is assigned the values from candidateSet 2 as the neural network defect. In step 15, the else condition represents if the $N N_{\text {Conf }}$ is not greater than or equal to $80 \%$. In step 16, candidate $_{\text {Final }}$ is assigned the values from candidateSet 1 as the computer 
vision defect. In step 17 , finally the hybrid algorithm returns the candidate $_{\text {Final }}$ as the final result of the WDD-DL system.

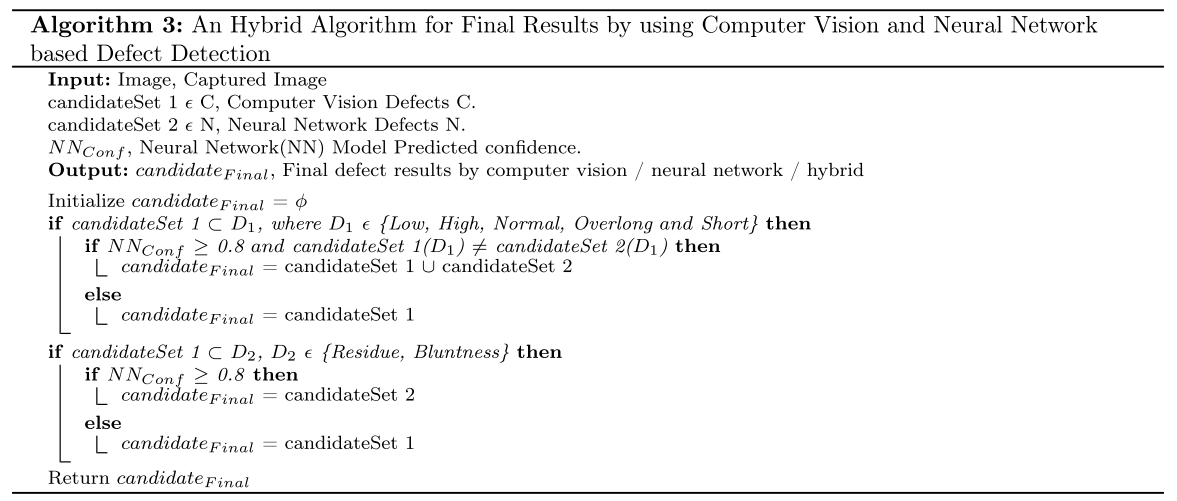

\section{Experiments}

For the experiments section, the workstation configuration used is presented in Table 2. All the experiments performed in this section are used to support proposed methodology demonstration of this paper. The results are evaluated using a large set of defect category and multiple defect category dataset using deep feature extraction. The system configuration consists of Solid State Drive (SSD) and Graphics Processing Unit (GPU) card for edge-glued wooden panel defect detection using deep feature extraction.

\section{Dataset}

The dataset given in Tables 3 and 4 presents the defect category and multiple defect category with their corresponding frames, respectively.

These two datasets are used for both training and testing with different ratios of CNN models for deep feature extraction. Various CNN models are used for checking the accuracy of the test dataset for selecting the high accuracy model and achieving best performance. The training data consist of various types of independent or combined edge-glued wooden defects, which is used to train a CNN model. A test data will then be used to check for the accuracy given by the various CNN models. All the data used for training and testing are taken from a single source Sakura furniture industry in Taichung, Taiwan. The high definition industrial camera is used for capturing different defect images to generate a dataset, which will be utilized by filters of computer vision (Kumar and Pang 2002b) 
Table 2 System configuration

\begin{tabular}{ll}
\hline Processor & Intel Core i7-7700 @ 3.60 GHz \\
\hline Memory & $16 \mathrm{~GB}$ \\
SSD card & Plextor PX-256S2C \\
Graphics card (GPU) & 1060 Nvidia GeForce GTX, 6GB \\
Python library & Numpy, Sklearn, OpenCV, Keras, \\
& Tensorflow, Matplotlib and \\
& Seaborn \\
\hline
\end{tabular}

Table 3 Dataset for defect category and frames

\begin{tabular}{llllll}
\hline Defect category & Normal & Bluntness & Residue & High & Low \\
\hline Frames & 1722 & 2343 & 1269 & 106 & 2655 \\
\hline
\end{tabular}

Table 4 Dataset for multiple defect category and frames

\begin{tabular}{llll}
\hline Defect category & Bluntness + Low & Residue + High & Residue + Low \\
\hline Frames & 525 & 1815 & 3 \\
\hline
\end{tabular}

and classification by CNN for evaluating the results. In Fig. 5a, the different category sample types are presented, which are used for this experimentation.

\section{Evaluations}

The configuration for CNN functional parameters is shown in Table 5, which presents the input parameters that are used for configuring the CNN model. The input shape denotes the height, width and depth (color channel) of the image used for processing. The batch size is used to indicate the data fitting in the batch of 10 to the network. A single epoch presents the dataset accessed in the set of batches within the neural network by a forward and backward pass at once, which is completed in iterations. The weights here are referred from "Imagenet" visual object recognition project (Russakovsky et al. 2015), which are learnable parameters used for controlling the signal within the network. The train and test ratio defines the distribution for the images into experiments for validation. Figure $5 \mathrm{~b}$ presents the output generated after processing images from Gabor filter. Four images are shown, which have bluntness defect type, given as input for the Gabor filter operation. The purpose of Gabor filter is to extract features of edge and perform the operation of convert scale, suitable for pattern identification. The result of two algorithms is similar, but the convert scale function has better identification evaluation.

In Fig. 6a, K-means, an unsupervised machine learning algorithm is used to find new pixel-based patterns by clustering, for image segmentation purpose. 

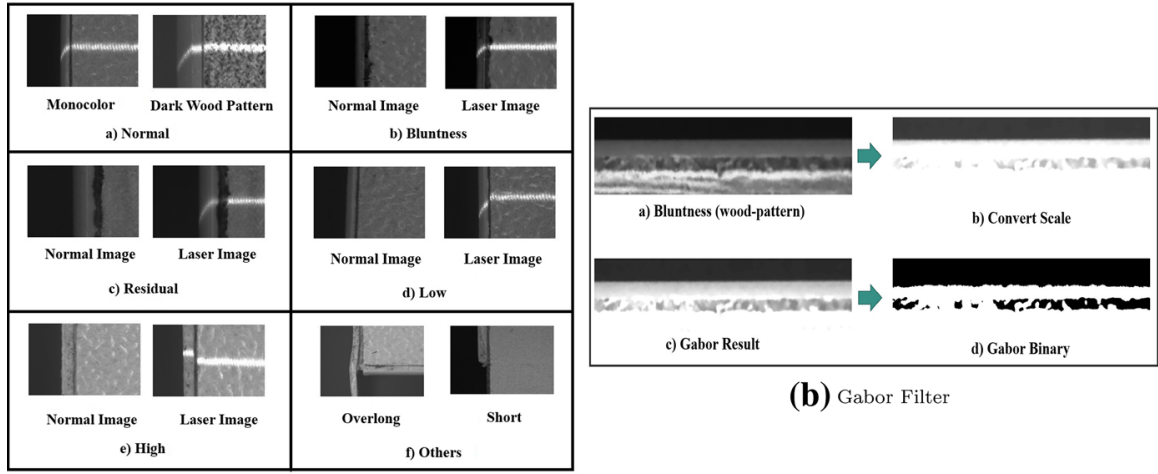

(b) Gabor Filter

(a) Defect Samples

Fig. 5 Different categories of defects and edge-glued defect evaluation

Table 5 Convolutional neural network $(\mathrm{CNN})$ parameters

\begin{tabular}{lllll}
\hline Input shape & Batch size & Epoch & Initial weights & Train and test ratio \\
\hline$(224,224,3)$ & 10 & 20 & Imagenet & $66: 33$ \\
\hline
\end{tabular}



(a) K-means and Find Contours

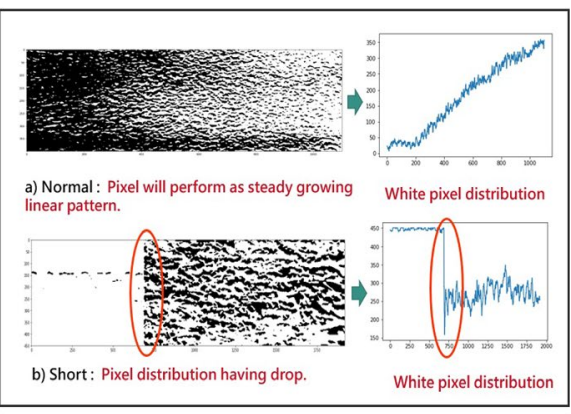

(b) Comparing Pixel Distribution

Fig. 6 Evaluation by basic image processing algorithms

Successively, Find contours function is used for defect detection within the input image for normal and bluntness types. Even though the output in processed image shows defect detection by red mark, it is still not evaluated to be effective for achieving high accuracy. In Fig. 6b, the pixel distribution comparison algorithm is used to capture and fetch the defect features and their positions, respectively. The high and low defect gap is used to represent the unnecessary gap between the tape and panel in the images. 


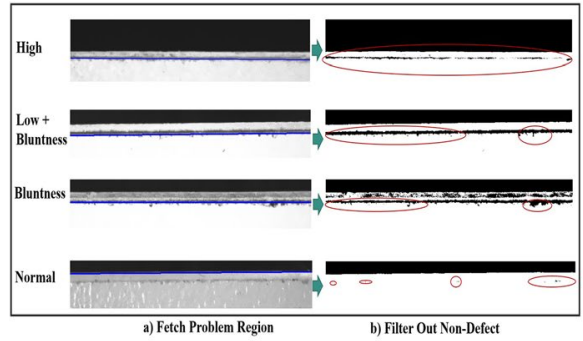

(a) Means Denoising and Threshold Otsu

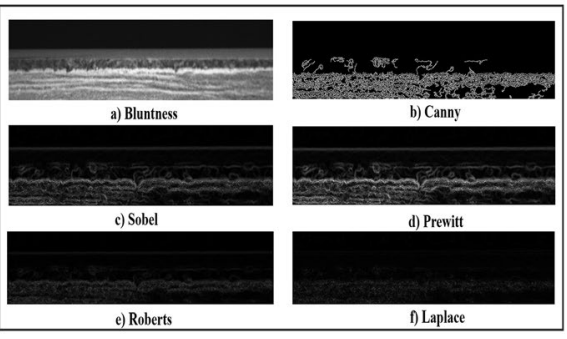

(b) Edge Detection

Fig. 7 Processing by denoising, threshold and edges

Therefore, the purpose of this algorithm is to extract the region, segments from the images with respect to foreground and background. It is still ineffective as per the expected results and demonstrated by the fluctuating graph.

In Fig. 7a, Means denoising and threshold otsu is used to present the algorithm for filtering out non-defect and fetch the defect regions after eliminating the noise. Henceforth, by a careful inspection, it was found to be suffering from similar accuracy issues as discussed previously for k-means. Figure $7 \mathrm{~b}$ shows various edge detection filtering algorithms such as Canny, Sobel, Prewitt, Roberts and Laplace, which are used to extract edge features from the input image. Each filter has its own way of edge feature extraction, which takes input of bluntness wood pattern type image.

Usually, applying computer vision-based filters are considered to be a basic process in edge defect detection. In Fig. 8, hybrid algorithms are used to present experiments by combining different algorithms. At first, the bluntness wooden pattern is taken as input. Here, applying smoothing and threshold algorithm before edge detection algorithm has achieved better detection results. The first

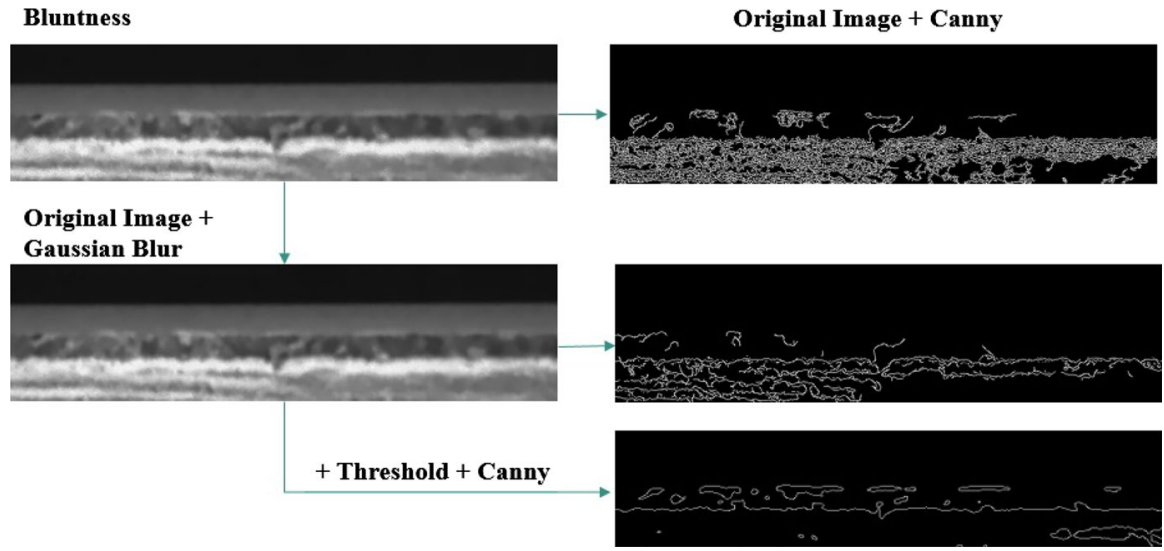

Fig. 8 Hybrid algorithm 


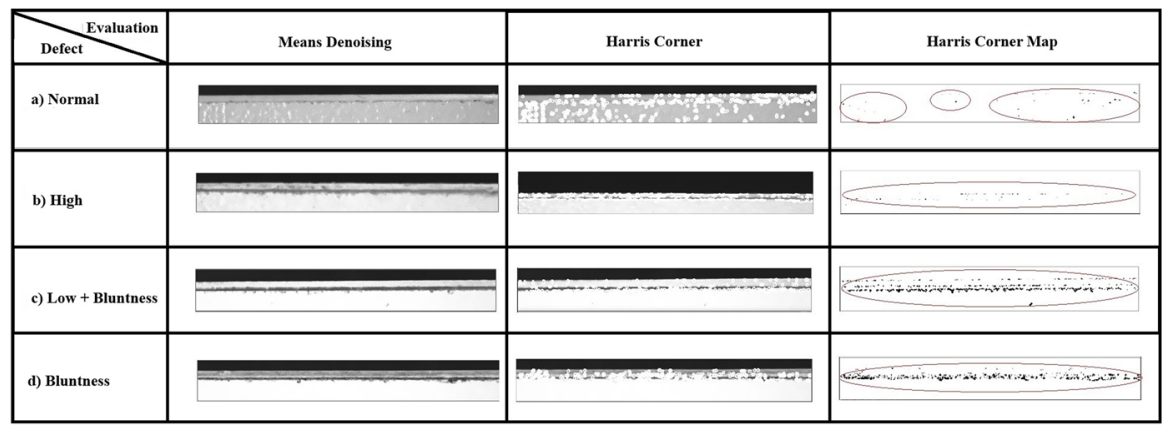

Fig. 9 Harris corner evaluations for different defect samples: a Normal, b High, c Low + Bluntness and d Bluntness

Table 6 Comparison of different defect classification models based on F1 score

\begin{tabular}{lllllll}
\hline Models/defects & Bluntness & Residue & High & Low & Normal & $\begin{array}{l}\text { Evalua- } \\
\text { tion time } \\
(\mathrm{s})\end{array}$ \\
\hline MobileNetV2 & 0.873 & 0.892 & 0.993 & 0.979 & 0.924 & $\mathbf{0 . 0 0 3}$ \\
ResNet50V2 & 0.625 & 0.844 & 0.995 & 0.667 & 0.83 & 0.005 \\
ResNet50 & 0.818 & 0.973 & $\mathbf{0 . 9 9 7}$ & 0.979 & 0.886 & 0.007 \\
DenseNet169 & $\mathbf{0 . 9 5}$ & 0.982 & $\mathbf{0 . 9 9 7}$ & 0.984 & $\mathbf{0 . 9 5 2}$ & 0.007 \\
InceptionResNetV2 & 0.904 & $\mathbf{0 . 9 8 6}$ & $\mathbf{0 . 9 9 7}$ & $\mathbf{0 . 9 9 1}$ & 0.93 & 0.008 \\
ResNet101V2 & 0.795 & 0.935 & 0.991 & 0.957 & 0.648 & 0.008 \\
ResNet101 & 0.736 & 0.735 & 0.86 & 0.761 & 0.61 & 0.009 \\
DenseNet201 & 0.703 & 0.974 & $\mathbf{0 . 9 9 7}$ & 0.984 & 0.903 & 0.009 \\
\hline
\end{tabular}

result is obtained after combining it with original image and canny. The second result is obtained by combining original and Gaussian blur. Similarly, third result is obtained by combining threshold with Canny. Figure 9 presents Harris corner evaluations for normal, high, low with bluntness and bluntness defect types. The process achieved by means denoising, Harris corner function and Harris corner map presents high accuracy evaluation of defects. Therefore, from Figs. 5b, 6, 7, 8, 9, Gabor function and Harris corner are only found to achieve high accuracy. Table 6 shows the F1 score calculated as a part of performance measure for the WDD-DL F1 score (also F-score or F-measure) as a measure of a test's accuracy. The column shows the various deep neural network models used within this paper for performing experiments, whereas the row for every model represents 5 types of defects detected from the wooden panel image by the respective neural network model. The values present the F1 score, which is given as a weighted average of precision and recall. 


$$
F 1 \text { Score }=2 \times \frac{\text { Precision } \times \text { Recall }}{\text { Precision }+ \text { Recall }}
$$

It is also known as an indicator to mark precision and recall together as given in Eq. (8). Here, the red colour highlighted values shows the highest score for a particular defect. In this case, the InceptionResNet v2 is the choice for this paper, as it shows the highest F1 score values evaluated among most of the defects in comparison. The defect detection time given by the InceptionResNet v2 is 0.008 sec, which is quite practical to present the results in the real time environment. The computer vision evaluation time for detecting a defect is given as 0.013, which is quite higher as compared to any of the deep neural network models listed above.

Figure 10 presents the performance measure of WDD-DL by precision, recall, F1 score and evaluation time for the different neural network models for the average of various types of defects. In Fig. 10a, the precision is used to present the actual "accuracy", when predicting the positive results. It can also be said as correctly predicted positive observations to the total predicted positive observations. The precision formula is given in Equation (9) as

$$
\text { Precision }=\frac{\text { TruePositive }(T P)}{\text { TruePositive }(T P)+\text { FalsePositive }(F P)}
$$

The highest precision in all deep neural network models is demonstrated by DenseNet 169 and InceptionResnet v2. Figure 10b presents the recall performance measure calculation for the actual positive output to "How much can be recalled",

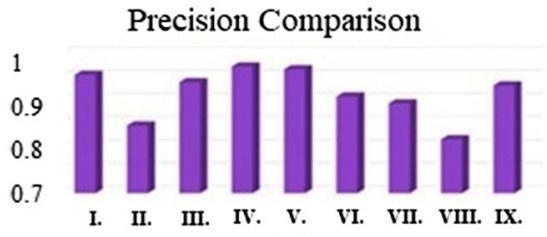

(a)

F1 Score Comparison

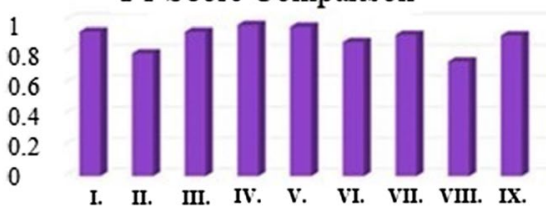

(c)

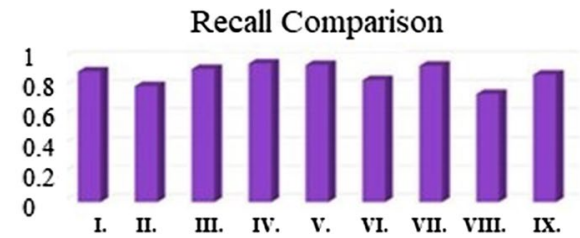

(b)

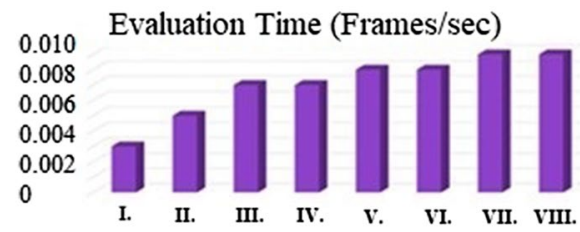

(d)

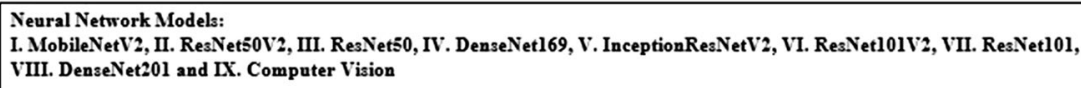

Fig. 10 Deep neural network performance measure comparison: a Precision, b Recall, c F1 Score and d Prediction Time 
when the actual situation is positive. Recall can be given as the ratio of correctly predicted positive observations over all observations in the actual class. The recall formula can be given as Eq. (10),

$$
\text { Recall }=\frac{\text { TruePositive }(T P)}{\text { TruePositive }(T P)+\text { FalseNegative }(F N)}
$$

The F1 score as presented before, is given in Fig. 10c where DenseNet 169 and InceptionResnet v2 are found to be leading in comparison to other deep neural network models. Figure 10d presents an evaluation time taken by each model for making a defect prediction. It can be observed that even though the fastest evaluation is performed by MobileNet v2, it does not provide higher F1 score for most of the different types of defect detection.

The benchmark results show the comparison between the ANN (DN) + Find contours method, LBP + SVM method, HOG+SVM method and WDD-DL system. Table 7 shows that the defects F1-score comparison is presented independently, recall is effective in the defect detection process, the precision for the alarm and evaluation time for the processing. Overall the WDD-DL system performs best in all the experiment cases for the performance and evaluation time to the recent references. The output processed image results and the detail benchmark results for the confusion matrix are given in Figure S1 and Figure S2 to S4 in Electronic Supplementary Material, respectively.

The highest recall is recorded by the DenseNet 196, DenseNet 201 and InceptionResnet v2 in all of the deep neural network models. The neural network model time is given by processing of 1 frame per second as prediction time. Even though the running time for half of the models shown in the table is less, the performance measure is taken as the priority. The performance measure given by accuracy is more instinctively accurate and can be given as the ratio of correctly predicted observations to the total observations.

Figure S5(a) in Electronic Supplementary Material, which shows Neural Network model training epoch vs. accuracy presents the increase in accuracy over more epochs until achieving stable accuracy rate, whereas Figure S5(b) in Electronic

Table 7 Benchmark comparison results for the defect classification

\begin{tabular}{|c|c|c|c|c|c|c|c|c|}
\hline Reference/algorithm & Bluntness & Residue & High & low & Normal & Recall & Precision & $\begin{array}{l}\text { Evaluation } \\
\text { time (sec.) }\end{array}$ \\
\hline $\begin{array}{l}\text { ANN (DN) + Find } \\
\text { Contours (Wenshu et al. } \\
2015)\end{array}$ & nan & 0.005 & 0.005 & nan & nan & 0.001 & 0.325 & 0.051 \\
\hline $\begin{array}{l}\text { LBP + SVM (Kuang } \\
\text { et al. 2018) }\end{array}$ & 0.275 & 0.707 & 0.909 & 0.655 & 0.455 & 0.489 & 0.908 & 108.5 \\
\hline $\begin{array}{l}\text { HOG + SVM (Sugiarto } \\
\text { et al. 2020) }\end{array}$ & 0.693 & 0.902 & 0.97 & 0.909 & 0.835 & 0.819 & 0.916 & 106.4 \\
\hline WDD-DL & 0.904 & 0.986 & 0.997 & 0.991 & 0.93 & 0.90 & 0.97 & 0.008 \\
\hline
\end{tabular}


Supplementary Material shows Neural Network model training epochs vs. loss presents the decrease in loss over more epochs until achieving stable loss rate.

\section{Overkill and escape analysis}

As the industrial measurement and inspection error are unavoidable, there are some issues faced that include defect-free cases, which are rejected (overkill) and defective cases are accepted (Escape) (Fu et al. 2011). Overkill and escape lead to high production cost and dissatisfaction of the customer, respectively. These overkill and escape analysis is used in industrial system for analysis and process improvement. The input parameters for obtaining the model in Figure S6 analysis of error distribution graph in Electronic Supplementary Material, is shown by Table 8 parameters set for overkill and escape analysis. The upper specification, the lower specification limit and average value are taken from the defect types low and high range. The total sigma is the process standard deviation, which is assumed to be normal distribution. The Gage R \& $\mathrm{R}$ (GRR) sigma is a measurement variation, which is narrowed as possible and taken between $10 \% \sim 30 \%$ as a marginal rate. Less than $10 \%$ are acceptable and less than 5\% is good but will incur high cost and hard to achieve. It can also be given as in Eq. (11),

Table 8 Parameters set for overkill and escape analysis

\begin{tabular}{llllll}
\hline USL & LSL & Average & Total sigma & GRR sigma & Bias \\
\hline 0.2 & -0.3 & -0.1 & 0.1 & 0.01 & -0.01 \\
\hline
\end{tabular}

Table 9 Parameters for GRR AND CPK

\begin{tabular}{lc}
\hline GRR (Percent of total variance) & $10 \%$ \\
\hline GRR (Percent tolerance with 5.15 distribution width) & $10 \%$ \\
Cpk Observed & 0.67 \\
Cpk Actual & 0.64 \\
\hline
\end{tabular}

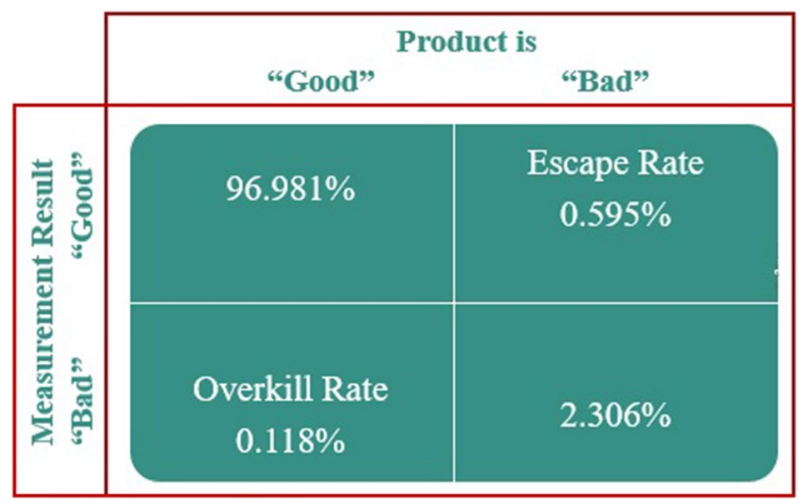

Fig. 11 The analysis of test cases 


$$
\text { GageR\&R }=\frac{5.15 \times \sigma_{m}}{U S L-L S L} \times 100 \%
$$

Here, the measurement system variation is $\sigma_{m}$, the upper specification limit is USL and the lower specification limit is LSL. The bias is the difference between observed mean and the reference value measurement, which can be positive or negative. As shown in Table $9, C_{p k}$ is the part of process capability index, which specifies predetermined specification limits for producing items that can be given as in Eq. (12),

$$
C_{p k}=\frac{\min (U S L-\mu, \mu-L S L)}{3 \sigma}
$$

Here, the process mean is $\mu$ and the process standard deviation is $\sigma$. Figure S6 in Electronic Supplementary Material, presents the analysis of error distribution, which shows the error range for USL and LSL. Variance and percentage of error is given as standard distribution, with low overkill rate and escape rate as tolerable values in process automation.

Figure 11 presents the analysis of test cases, which can be stated as:

1. A good part is evaluated to be good.

2. A bad part is evaluated to be bad.

3. (Type I error) Overkill: A good part is evaluated to be bad.

4. (Type II error) Escape: A bad part is evaluated as good.

The observations from the measurement system for fault rate analysis is when we increase sigma gradually, the overkill rate and escape rate are found to be increasing. In case of increasing GRR sigma, the overkill rate is increasing, but the escape rate is found to be fluctuating highly, whereas for increasing the bias, the overkill rate and escape rate are increasing too. Higher accuracy in defect detection can be achieved, which will require hyper-parameter setting in deep neural network and will take high processing power for evaluation.

\section{Discussion}

\section{Analysis of CNN model and evaluation}

Convolutional Neural Network (CNN) serves the purpose of feature extraction and classification. During the baseline $\mathrm{CNN}$ evaluation, several challenges are faced with different angles (rotation, translation and scaling), different background and illumination. In such case, high training images of the defect are required, which is quite a challenge for identifying different shapes and sizes of defect. To overcome these challenges, we have used geometric filters to capture different category of defect features as for two-stage classification. The illumination problem was solved using batch normalization and rectified linear unit (ReLU). CNN is usually stuck in local minima because of high redundancy in training feature. To avoid this problem, 
feature selection used within the algorithm helps to resolve such issue. Still, evaluation metrics in CNN was average for accuracy, precision, recall and F1-score because of over-fitting. Therefore, optimization was needed to achieve best prediction without over-fitting. The over-fitting problem is resolved by using dropout layer and ReLU as a part of regularization. Also applying minimal CNN and some well known CNN models was not enough. To achieve better performance, more experiments and high-end GPU for processing were used. InceptionResnet v2 performed better than other CNN models by having high accuracy and recall with better evaluation time for different defect detection models.

For baseline CNN, the training time is high, whereas classification is average, there is a need of proper data pre-processing, CNN layer configuration and feature extraction. The CNN performed with high accuracy in capturing different defect shapes and sizes with geometric filters. Feature extraction was also simplified by categorizing different types of defects distinctly. Even though geometric filters were applied, most of the CNN models suffered in accuracy because of high false alarm rate. Loss incurred is higher too, while performing convolution operation. A proper setting for CNN configuration is one of the crucial tasks for achieving better performance with minimal values as shown in Table 5 (CNN parameters). In addition, the CNN with appropriate filters and multiple planned experiments constitutes a finely executed approach for implementing effective and efficient research. $\mathrm{CNN}$ is incomplete without the support of high-end GPU hardware to accelerate the implementation.

\section{Structural light detection for the depth edges}

The purpose of using structured light detection is to perform depth edges detection accurately and avoid scattering of the inner texture edges. Usually, the depth discontinuities based distortion depends upon the distance from the camera (Park et al. 2008). The depth edge detection is crucial in the defect detection process as it represents object contours. The limitations of the traditional computer vision-based edge detection method are no differentiation present within depth edges and texture edges. Moreover, detecting depth edges is possible by eliminating the need of reconstruction of dense 3D image scenes. The process of detecting depth edge map consists of consecutively projecting white and structured light of the target object. The pattern image is obtained by differentiating the white and structured light images with robust threshold criteria. The final depth edge map is achieved by capturing candidate locations from the Gabor filter by identifying depth edge and distortions of the light patterns.

\section{Experience sharing for development and deployment}

Wooden furniture has several applications in the market including homes, offices, shops, restaurants, etc. Several manufacturing industries are completely dependent on the human workers for identification of various types of defects. In such cases, the wood material is first cut into a wooden panel, which is then sent for the 
edge-glued banding process. During the edge-glued banding process, several defects can arise that include tape can be high, low, bluntness, residue, short and overlong. Traditionally, a quality check (Q.C.) sticker was put on the wooden panel after the human worker inspection. There are high chances that the human identification of such defects can be missed. In addition, the human worker needs extra time other than the edge banding process to identify the defects because missing an error may affect the brand image and quality of the furniture product. Due to inefficiency of this process, WDD-DL was designed to overcome this problem and provide realtime identification of edge-glued banding defects. At the start of WDD-DL, some basic image filters were used to identify such defects but due to their inaccuracy only Gabor and Harris corner were used with a deep feature extraction as a two stage model to achieve higher accuracy.

Comparing the literature (Hao et al. 2020) and this paper's experiments, most of the systems use the same group of edge feature detection filters. WDD-DL experiments have been performed on various filters including the hybrid ones but demonstrate better results with the use of red line laser with filtering and different performance evaluation with accuracy and loss. Various defect/data feature collections were performed with infrared, ccd cameras but was decided to use line laser as to represent the highlight of the model. The edge-glued based defect detection can be performed on wooden panels of different shapes and sizes. The image quality is controlled by adjusting the angle of the red line laser (laser-aligned), frequency of taking images is maintained to 75 frames/sec by the use of practical industrial camera in the experimentation environment. The pre-processing of the image taken to get the necessary features includes specifying the image resize and several defect labelling to the training images with the help of manual operations too. The speed of the conveyor is not an issue as the 75 frames/sec are taken by the camera to process the frame. Documentation of the production operation can be done by generating a report that includes day-wise, weekly or monthly production with average errors encountered per day, per week or per month respectively. The WDD-DL system shows very few false alarm detected as the accuracy presented by the CNN models is quite high. The threshold set by the CNN model is $80 \%$ for identifying a defect type based on the training defect images. Ultimately, the human operators sort out the defective wooden panels after edge banding based on the error alert as shown by the WDD-DL display screen. The WDD-DL system performs comparison with different edge defect detection algorithms, CNN models and evaluates them based on F1 score, precision, recall, accuracy and loss. Henceforth, the overall benefit of the system can be represented as high accuracy, real-time defect detection, while the wooden panel is on the conveyor after the edge banding process.

\section{Applying AOI in the industrial environment}

AOI approach is being applied to solve many industrial challenges (He et al. 2019b; Block et al. 2020). It is focused on reducing human dependency, improve speed and achieve high accuracy. The use of AOI has helped the quality control process to be transformed and implement process automation to identify, notify, alert and display 
the affected region. The purpose of using AOI is to provide high accuracy defect detection for quality inspection and higher production with less maintenance cost. The performance modelling can be done by accuracy, precision, F1-score and recall from the confusion matrix calculations.

The deep learning algorithms are well known to provide only classification, which is insufficient to locate exact defect position on the edge-glued wooden panel. Therefore, a two-stage approach consists of high level pre-processing by computer vision that captures laser based non-segmented defects for size of defect and type stored in candidate set 1 , whereas the other defect patterns detected by better classification are stored in candidate set 2 . Later, both candidate set 1 and candidate set 2 are combined to represent as a complete final result. "The computer vision based filtering consists of a rule-based system for achieving good accuracy results, whereas the deep learning based classification provides the enhancement for the best accuracy." Ultimately, the innovation statement can be stated as the various wood-based edge-glued defect detection in the WDD-DL having structured light detection and line laser (multi-sensors) by using the computer vision-based pre-processing, filtering and convolutional neural networks for re-evaluation, to achieving high accuracy results. The goal of this paper is to perform hardware and software based integration using AOI and to share practical industrial implementation experience for achieving high accuracy results.

\section{Limitations of the WDD-DL}

Limitation of this work can be given that the experiments are performed only on 21 different wood types, and fewer defect training images were available for overlong and short edge banding. However, the improvements are not a big challenge to be implemented as only some additional training is required for the CNN model. The approach presented by the WDD-DL paper is multi-disciplinary research. This paper is the successor for the edge based defect detection process in the furniture industry. In the future, WDD-DL can be included as a part of the ERP system operating within the respective industrial environment to have better track of Quality Control (Q.C.) process and records for reporting.

\section{Conclusion}

WDD-DL provides automation for the development of the production line, by easing defect detection work load on the human machine operators. Integration of WDD-DL and production line helps to complete the workflow automation process. Edge-glued wooden defect detection has high demand in the furniture industry, having products for homes, offices, shops, institutions, organizations, etc. Therefore, advancing the real-time defect detection process, which is the part of quality control in the industrial environment by using various computer vision and $\mathrm{CNN}$ algorithms for achieving higher accuracy. The use of two-stage model utilizing computer 
vision-based filtering and deep learning-based classification by using InceptionResnetV2 is found to be yielding best performance with the highest recall of 0.90 in comparison with other popular CNN models, which is effective for the defect detection process. Moreover, the evaluation time is quite reasonable to be shown on the live screen at 0.008 seconds. The outcome of this paper can be given as achieving high accuracy quality control in the furniture industry using multi-sensor, twostage approach for real-time monitoring of defects. The paper also provides detailed overkill and escape rate analysis. The discussion section containing interaction with industrial employees after implementing the WDD-DL provides various insights into the practical working scenario. In the future work, a new interpretable AI model is planned to be demonstrated, which can detect different kinds of defects including the wooden panel surface defects for overall industrial product defect inspection. Ultimately, the paper achieves better performance for the quality control process by WDD-DL with higher F1 score.

Supplementary Information The online version contains supplementary material available at https://doi. org/10.1007/s00226-021-01316-3.

Acknowledgements This research is funded by the DDS-THU AI Center of Tunghai University, Taiwan and Taiwan Sakura Corporation with the project code: 108670. Also a special thanks to the funding from ministry of science and technology (MOST), Taiwan with the project code: "MOST 109-2622-E-029013-", reviewers and editors of the WSAT journal.

\section{Declarations}

Conflict of interest The authors declare that they have no conflict of interest.

Open Access This article is licensed under a Creative Commons Attribution 4.0 International License, which permits use, sharing, adaptation, distribution and reproduction in any medium or format, as long as you give appropriate credit to the original author(s) and the source, provide a link to the Creative Commons licence, and indicate if changes were made. The images or other third party material in this article are included in the article's Creative Commons licence, unless indicated otherwise in a credit line to the material. If material is not included in the article's Creative Commons licence and your intended use is not permitted by statutory regulation or exceeds the permitted use, you will need to obtain permission directly from the copyright holder. To view a copy of this licence, visit http://creativecommons.org/licen ses/by/4.0/.

\section{References}

Abdullah ND, Hashim UR, Ahmad S, Salahuddin L (2020) Analysis of texture features for wood defect classification. Bul Electr Eng Inf 9(1):121-128

Akiba T, Suzuki S, Fukuda K (2017) Extremely large minibatch sgd: training resnet-50 on imagenet in 15 minutes. arXiv preprint arXiv:1711.04325

Aleksi I, Sušac F, Matić T (2019) Features extraction and texture defect detection of sawn wooden board images. In: 2019 27th Telecommunications Forum (TELFOR), IEEE, pp 1-4

Block SB, da Silva RDD, Dorini L, Minetto R (2020) Inspection of imprint defects in stamped metal surfaces using deep learning and tracking. IEEE Trans Ind Electron. https://doi.org/10.1109/TIE.2020.2984453

Cao J, Zhang J, Wen Z, Wang N, Liu X (2017) Fabric defect inspection using prior knowledge guided least squares regression. Multimed Tools Appl 76(3):4141-4157

Chang Z, Cao J, Zhang Y (2018) A novel image segmentation approach for wood plate surface defect classification through convex optimization. J For Res 29(6):1789-1795 
Chen H, Hu Q, Zhai B, Chen H, Liu K (2020) A robust weakly supervised learning of deep conv-nets for surface defect inspection. Neural Comput Appl 32:11229-11244

Chen N, Men X, Han X, Wang X, Sun J, Chen H (2018) Edge detection based on machine vision applying to laminated wood edge cutting process. In: 2018 13th IEEE conference on industrial electronics and applications (ICIEA), IEEE, pp 449-454

Contreras Masse R (2019) Application of iot with haptics interface in the smart manufacturing industry. Instituto de Ingenierı y Tecnologia

Czimmermann T, Ciuti G, Milazzo M, Chiurazzi M, Roccella S, Oddo CM, Dario P (2020) Visualbased defect detection and classification approaches for industrial applications-a survey. Sensors 20(5):1459

Derpanis KG (2004) The harris corner detector. York University, 2

Fang Y, Lin L, Feng H, Lu Z, Emms GW (2017) Review of the use of air-coupled ultrasonic technologies for nondestructive testing of wood and wood products. Comput Electron Agric 137:79-87

Fu S, Kauppila O, Mottonen M (2011) Measurement system escape and overkill rate analysis. Int J Adv Manuf Technol 57(9-12):1079

Gao Y, Gao L, Li X, Wang XV (2019) A multilevel information fusion-based deep learning method for vision-based defect recognition. IEEE Trans Instrum Meas 69(7):3980-3991

Hao R, Lu B, Cheng Y, Li X, Huang B (2020) A steel surface defect inspection approach towards smart industrial monitoring. J Intell Manuf 32:1833-1843

Hashim UR, Hashim SZM, Muda AK (2016) Performance evaluation of multivariate texture descriptor for classification of timber defect. Optik 127(15):6071-6080

He K, Zhang X, Ren S, Sun J (2016) Deep residual learning for image recognition. In: Proceedings of the IEEE conference on computer vision and pattern recognition, pp 770-778

He T, Liu Y, Xu C, Zhou X, Hu Z, Fan J (2019) A fully convolutional neural network for wood defect location and identification. IEEE Access 7:123453-123462

He T, Liu Y, Yu Y, Zhao Q, Hu Z (2020) Application of deep convolutional neural network on feature extraction and detection of wood defects. Measurement 152:107357

He Y, Song K, Meng Q, Yan Y (2019) An end-to-end steel surface defect detection approach via fusing multiple hierarchical features. IEEE Trans Instrum Meas 69(4):1493-1504

Hu J, Song W, Zhang W, Zhao Y, Yilmaz A (2019) Deep learning for use in lumber classification tasks. Wood Sci Technol 53(2):505-517

Kuang H, Ding Y, Li R, Liu X (2018) Defect detection of bamboo strips based on lbp and glcm features by using svm classifier. In: 2018 Chinese control and decision conference (CCDC), IEEE, pp 3341-3345

Kumar A, Pang GK (2002a) Defect detection in textured materials using gabor filters. IEEE Trans Ind Appl $38(2): 425-440$

Kumar A, Pang GK (2002b) Defect detection in textured materials using optimized filters. IEEE Trans Syst Man Cybern Part B (Cybernetics) 32(5):553-570

Li C, Zhang Y, Tu W, Jun C, Liang H, Yu H (2017) Soft measurement of wood defects based on lda feature fusion and compressed sensor images. J For Res 28(6):1285-1292

Li S, Li D, Yuan W (2019) Wood defect classification based on two-dimensional histogram constituted by lbp and local binary differential excitation pattern. IEEE Access 7:145829-145842

Mu H, Zhang M, Qi D, Ni H (2015) The application of rbf neural network in the wood defect detection. Int J Hybrid Inf Technol 8(2):41-50

Park J, Kim C, Na J, Yi J, Turk M (2008) Using structured light for efficient depth edge detection. Image Vision Comput 26(11):1449-1465

Rahiddin RNN, Hashim UR, Ismail NH, Salahuddin L, Choon NH, Zabri SN (2020) Classification of wood defect images using local binary pattern variants. Int J Adv Intell Inform 6(1):36-45

Russakovsky O, Deng J, Su H, Krause J, Satheesh S, Ma S, Bernstein M et al (2015) Imagenet large scale visual recognition challenge. Int J Comput Vis 115(3):211-252

Sindagi VA, Srivastava S (2015) Oled panel defect detection using local inlier-outlier ratios and modified lbp. In: 2015 14th IAPR international conference on machine vision applications (MVA), IEEE, pp 214-217

Song W, Chen T, Gu Z, Gai W, Huang W, Wang B (2015) Wood materials defects detection using image block percentile color histogram and eigenvector texture feature. In: First international conference on information sciences, machinery, materials and energy, Atlantis Press, pp 779-783

Sugiarto B, Arifin MR, Laluma RH, Prakasa E, Azwar AG et al (2020) An improved wood identification accuracy using gaussian pyramid and laplacian edge detection based on android smartphone. In: 2020 14th international conference on telecommunication systems, services, and applications (TSSA), IEEE, pp 1-5 
Thumm A, Riddell M (2017) Resin defect detection in appearance lumber using $2 \mathrm{~d}$ nir spectroscopy. Eur J Wood Wood Prod 75(6):995-1002

Tong H, Ng H, Yap T, Ahmad W, Fauzi M (2017) Evaluation of feature extraction and selection techniques for the classification of wood defect images. J Eng Appl Sci 12(3):602-608

Urbonas A, Raudonis V, Maskeliūnas R, Damaševičius R (2019) Automated identification of wood veneer surface defects using faster region-based convolutional neural network with data augmentation and transfer learning. Appl Sci 9(22):4898

Wang J, Li Q, Gan J, Yu H, Yang X (2019) Surface defect detection via entity sparsity pursuit with intrinsic priors. IEEE Trans Ind Inform 16(1):141-150

Wells L, Gazo R, Del Re R, Krs V, Benes B (2018) Defect detection performance of automated hardwood lumber grading system. Comput Electron Agric 155:487-495

Wen W, Xia A (1999) Verifying edges for visual inspection purposes. Pattern Recognit Lett 20(3):315-328

Wenshu L, Lijun S, Jinzhuo W (2015) Study on wood board defect detection based on artificial neural network. Open Autom Control Syst J. https://doi.org/10.2174/1874444301507010290

Yang H, Yu L (2017) Feature extraction of wood-hole defects using wavelet-based ultrasonic testing. J For Res 28(2):395-402

Yun JP, Lee SJ, Koo G, Shin C, Park C (2019) Automatic defect inspection system for steel products with exhaustive dynamic encoding algorithm for searches. Opt Eng 58(2):023107

Zhang Y, Davison BD (2019) Modified distribution alignment for domain adaptation with pre-trained inception resnet. arXiv preprint arXiv:1904.02322

Publisher's Note Springer Nature remains neutral with regard to jurisdictional claims in published maps and institutional affiliations.

\section{Authors and Affiliations}

\section{Lun-Chi Chen ${ }^{1}$ Mayuresh Sunil Pardeshi ${ }^{2} \cdot$ Win-Tsung Lo ${ }^{1,2} \cdot$ Ruey-Kai Sheu $^{1}$ (1). Kai-Chih Pai ${ }^{1} \cdot$ Chia-Yu Chen ${ }^{1} \cdot$ Pei-Yu Tsai ${ }^{1} \cdot$ Yueh-Tiann Tsai ${ }^{3}$}

Ruey-Kai Sheu

rickysheu@thu.edu.tw

1 Department of Computer Science, Tunghai University, Taichung City, Taiwan (R.O.C.)

2 DDS-THU AI Center, Tunghai University, Taichung City, Taiwan (R.O.C.)

3 Taiwan Sakura Corporation, Taichung City, Taiwan (R.O.C.) 